-研究报告・

\title{
中国西南地区重要木本油料植物扁核木的遗传结 构及成因
}

叶俊伟(D1,2, 田斌 (D) ${ }^{*}$

1. 西南林业大学国家林业局西南地区植物多样性保育重点实验室, 昆明 650224; 2. 中国科学院昆明植物研究所中国西南野生生物种质资 源库, 昆明 650201

摘要: 扁核木(Prinsepia utilis)为中国西南地区温带森林重要的木本油料植物, 但对其野生资源种群遗传结构及成因的了解严 重不足。我们采用核微卫星分子标记, 对32个扁核木自然种群共377个个体的群体演化历史进行了探讨, 并评估其遗传资源。 研究发现扁核木种群自西向东可划分 4 个遗传群组, 即喜马拉雅、横断山以及云贵高原西部和东部群组。其中, 最大的遗传分 化存在于喜马拉雅和其他区域种群间。与喜马拉雅和云贵高原东部群组相比, 横断山和云贵高原西部群组混合了其他群组的 遗传成分。种群动态历史分析显示中部 2 个群组在喜马拉雅和云贵高原东部群组形成后形成, 不同群组间的分化均发生在更 新世晚期。地理隔离和环境隔离分析表明扁核木种群间的遗传分化主要由环境差异导致。环境差异分析显示不同群组间的气 候存在不同程度的差异，其中喜马拉雅和云贵高原东部群组与中部2个群组间的差异显著。此外，结合该物种不同时期的生态 位模拟数据, 我们认为喜马拉雅和云贵高原地区的遗传资源在未来需要优先保护。

关键词: 核微卫星; 遗传多样性; 主成分分析; 喜马拉雅

叶俊伟, 田斌 (2021) 中国西南地区重要木本油料植物扁核木的遗传结构及成因. 生物多样性, 29, 1629-1637. doi: 10.17520/biods.2021341. Ye JW, Tian B (2021) Genetic structure and its causes of an important woody oil plant in Southwest China, Prinsepia utilis (Rosaceae). Biodiversity Science, 29, 1629-1637. doi: 10.17520/biods.2021341.

\section{Genetic structure and its causes of an important woody oil plant in Southwest China, Prinsepia utilis (Rosaceae)}

Junwei Ye ${ }^{(\mathbb{D} 1,2}$, Bin $\operatorname{Tian}^{(\mathbb{D} 1 *}$

1 Key Laboratory of Biodiversity Conservation in Southwest China, State Forestry Administration, Southwest Forestry University, Kunming 650224

2 Germplasm Bank of Wild Species in Southwest China, Kunming Institute of Botany, Chinese Academy of Sciences, Kunming 650201

\footnotetext{
ABSTRACT

Aims: Prinsepia utilis is an important woody oil species in temperate forests in Southwest China, however, almost nothing is known about its population genetic structure and the causes. So, we aim to study its evolutionary history through multiple nuclear microsatellite loci (nSSRs).

Methods: We sampled 377 individuals from 32 natural populations across all distribution range of $P$. utilis. All individuals were amplified and scored using seven self-developed nSSRs markers. Genetic structure was inferred using STRUCTURE software and principal component analysis. The most possible demographic scenario and corresponding parameters were modeled and estimated in DIYABC. Genetic diversity and genetic differentiation of each population, locus and genetic cluster were calculated. Contributions of geographic distance and environmental differences to genetic differentiation were calculate through Mantel test and partial Mantel test. At last, environmental differences among different genetic clusters was evaluated through principal component analysis using 19 climatic variables.

Results: All population can be divided into four genetic groups that are Himalayas, Hengduan Mountains, west and east

收稿日期: 2021-08-30; 接受日期: 2021-11-19

基金项目: 云南省高层次人才培养支持计划“青年拔尖人才”专项(YNWR-QNBJ-2020)、国家自然科学基金(41861008)和云南省教育厅科学研究基金 (2018JS347)

* 通讯作者 Author for correspondence. E-mail: tianbin@swfu.edu.cn
} 
Yunnan-Guizhou Plateau genetic groups from west to east. The greatest genetic differentiation occurs between populations in the Himalayas and other regions. In comparison with the Himalayan and east Yunnan-Guizhou Plateau groups, the Hengduan Mountains and west Yunnan-Guizhou Plateau groups have higher proportion of genetic mixture from other groups. Population demographic history analysis indicate two central groups are formed after Himalayas and east Yunnan-Guizhou Plateau groups, all divergences are occurred in late Pleistocene. The analyses of isolation by distance and isolation by environment show that the genetic differentiation of $P$. utilis is mainly caused by environmental difference. Environmental difference analysis using 19 climatic variables shows various difference among groups with significant difference exist between Himalayas and east Yunnan-Guizhou Plateau groups.

Conclusion: The distinct genetic structure of $P$. utilis is formed through the combination of Pleistocene climatic changes, complicated environment and its own biological characters. Combined with ecological niche modeling analysis in different periods, we suggest protection of genetic resources in Himalayas and Yunnan-Guizhou Plateau should be first priority.

Key words: nuclear microsatellites; genetic diversity; principal component analysis; Himalayas

中国西南地区是重要的生物多样性热点地区 (李锡文和李捷, 1993; Sun et al, 2017), 丰富的历史 地质活动和气候变化以及多样的地形环境条件为 植物物种的形成、分化和保存提供了大量的机会 (Sun et al, 2017; Muellner-Riehl, 2019; Ding et al, 2020), 使其成为生物多样性的“摇篮”和 “博物馆” (Xing \& Ree, 2017; Lu et al, 2018)。

谱系地理学研究表明地理隔离和环境差异是 中国西南地区生物类群种群分化的重要驱动力。其 中高山峡谷地形是重要的地理隔离(刘杰等, 2017), 促进了不同地理种群间的异域分化, 尤其是怒江一 澜沧江分水岭(Mekong-Salween Divide, MSD)造成 了明显的谱系地理间断(Ward, 1921; 叶俊伟等, 2017)。此外, “空中岛屿”间低海拔的生境也能构成 扩散障碍, 限制不同 “岛屿”群体间的基因流, 形成 显著的遗传分化(Luo et al, 2016)。环境差异同样可 以促使不同区域的种群间产生适应性分化(Liu et al, 2013; Ma et al, 2019), 导致明显的遗传结构。因此, 中国西南地区拥有丰富的物种多样性和遗传资源 (Sun et al, 2017)。

扁核木(Prinsepia utilis)是蓄薇科东亚特有属扁 核木属的多年生常绿灌木, 主要分布于中国西南的 喜马拉雅、横断山及云贵高原地区。扁核木为温带 森林重要的木本油料植物(出油率为 $30 \%$ 左右), 平 均盛果期可达 40 年, 有较高的食用、保健和药用价 值(Wang et al, 2013)。此外, 扁核木适应能力和再生 能力强、根系发达, 可绿化荒山(和琼姬等, 2016), 有重要生态价值。但是, 国内外对扁核木的研究多 集中于其不同部位化学物质的提取、鉴定和理化活 性功能的验证方面(Guan et al, 2013, 2014; Huang et al, 2017)。对这一重要经济木本植物遗传资源的调 查和评估严重缺乏, 仅有Wang等(2013)报道了其叶 绿体基因组序列和变异, 以及 $\mathrm{Ma}$ 等(2019)和Jin等 (2020)对扁核木所在扁核木属开展的生物地理学研 究。随着农业生产的快速增长和野外资源的大量采 集，扁核木种群破碎化日趋严重。因此，我们基于 覆盖扁核木全分布区的种群采集，通过多位点的核 微卫星数据, 拟厘清其种群的遗传结构和成因以及 需要重点保护和进行种质资源收集的地理区域。

\section{材料与方法}

\section{1 样品采集和DNA提取}

2015-2017年, 我们对扁核木32个种群的377个 野生成年个体进行了采集(表1), 同一种群不同个 体的间距至少为 $30 \mathrm{~m}$ 。新鲜叶片样品采集后经硅胶 干燥保存。凭证标本保存于西南林业大学国家林业 局西南地区生物多样性保育重点实验室。所有样品 的DNA用改良的CTAB法(Doyle \& Doyle, 1990)提 取。

\section{2 核微卫星引物开发、篮选、扩增和测序}

基于扁核木转录组开发可用的核微卫星引物 (Ju et al, 2015)。微卫星扩增的 $25 \mu \mathrm{L}$ PCR反应体系 中包含 $1 \mu \mathrm{L}$ DNA, $12.5 \mu \mathrm{L} 2 \times$ Taq MasterMix

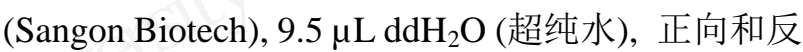
向引物各 $1 \mu \mathrm{L}$ 。扩增程序如下: 94C 预变性5 min; $94^{\circ} \mathrm{C}$ 变性 $30 \mathrm{~s}$, 退火45 s, 退火温度根据引物调整 (附录1), $72^{\circ} \mathrm{C}$ 延伸 $1 \mathrm{~min}$, 进行30次循环; 最后 $72^{\circ} \mathrm{C}$ 延伸 $10 \mathrm{~min}$ 。完成后在 $4^{\circ} \mathrm{C}$ 保存。通过不同区域 4 个 种群的 8 个个体进一步篮选电泳条带单一清晰无杂 带及多态性高易读取的引物。在微卫星引 
表1 扁核木种群取样和遗传多样性信息

Table 1 Sampling and genetic diversity information of Prinsepia utilis

\begin{tabular}{|c|c|c|c|c|c|c|c|c|c|c|}
\hline $\begin{array}{l}\text { 代号 } \\
\text { Code }\end{array}$ & $\begin{array}{l}\text { 地点 } \\
\text { Site }\end{array}$ & $\begin{array}{l}\text { 经纬度 } \\
\text { Location }\end{array}$ & $\begin{array}{l}\text { 海拔 } \\
\text { Altitude (m) }\end{array}$ & $n$ & $A_{0}$ & $H_{\mathrm{E}}$ & $H_{\mathrm{O}}$ & $R_{\mathrm{S}}$ & $P_{\mathrm{AR}}$ & $F_{\text {IS }}$ \\
\hline BAZY & 云南昆明 Kunming, Yunnan & $102.72^{\circ} \mathrm{E}, 25.40^{\circ} \mathrm{N}$ & 2,370 & 9 & 20 & 0.45 & 0.63 & 2.45 & 0.01 & -0.35 \\
\hline BBM & 西藏波密 Bomi, Xizang & $95.34^{\circ} \mathrm{E}, 29.99^{\circ} \mathrm{N}$ & 2,750 & 14 & 22 & 0.54 & 0.76 & 2.61 & 0 & -0.37 \\
\hline BDC & 四川稻城 Daocheng, Sichuan & $100.30^{\circ} \mathrm{E}, 28.58^{\circ} \mathrm{N}$ & 3,310 & 12 & 21 & 0.46 & 0.62 & 2.55 & 0.12 & -0.31 \\
\hline BDCQ & 云南会泽 Huize, Yunnan & $103.10^{\circ} \mathrm{E}, 25.94^{\circ} \mathrm{N}$ & 2,220 & 10 & 22 & 0.48 & 0.67 & 2.74 & 0.07 & -0.35 \\
\hline BES & 云南峨山 E’shan, Yunnan & $102.11^{\circ} \mathrm{E}, 24.28^{\circ} \mathrm{N}$ & 2,060 & 6 & 15 & 0.38 & 0.62 & - & - & -0.58 \\
\hline BHD & 四川会东 Huidong, Sichuan & $102.78^{\circ} \mathrm{E}, 26.55^{\circ} \mathrm{N}$ & 2,370 & 14 & 22 & 0.47 & 0.65 & 2.61 & 0 & -0.36 \\
\hline BHQ & 云南鹤庆 Heqing, Yunnan & $100.18^{\circ} \mathrm{E}, 26.56^{\circ} \mathrm{N}$ & 2,200 & 15 & 27 & 0.64 & 0.86 & 3.28 & 0 & -0.31 \\
\hline BJL & 西藏吉隆 Jilong, Xizang & $85.36^{\circ} \mathrm{E}, 28.39^{\circ} \mathrm{N}$ & 3,140 & 12 & 19 & 0.46 & 0.65 & 2.40 & 0.03 & -0.38 \\
\hline BJP & 云南澄江 Chengjiang, Yunnan & $102.86^{\circ} \mathrm{E}, 24.73^{\circ} \mathrm{N}$ & 2,180 & 15 & 25 & 0.58 & 0.81 & 2.93 & 0 & -0.38 \\
\hline BLD & 云南玉龙 Yulong, Yunnan & $99.46^{\circ} \mathrm{E}, 27.18^{\circ} \mathrm{N}$ & 2,630 & 16 & 21 & 0.56 & 0.88 & 2.59 & 0 & -0.54 \\
\hline BLJ & 云南丽江 Lijiang, Yunnan & $100.23^{\circ} \mathrm{E}, 27.18^{\circ} \mathrm{N}$ & 3,650 & 5 & 19 & 0.52 & 0.78 & - & - & -0.47 \\
\hline BLJS & $\begin{array}{l}\text { 云南老君山 Laojun Mountains, } \\
\text { Yunnan }\end{array}$ & $103.93^{\circ} \mathrm{E}, 23.30^{\circ} \mathrm{N}$ & 1,640 & 15 & 25 & 0.55 & 0.84 & 2.83 & 0 & -0.51 \\
\hline BLJW & 贵州安顺 Anshun, Guizhou & $105.94^{\circ} \mathrm{E}, 26.25^{\circ} \mathrm{N}$ & 1,400 & 15 & 23 & 0.49 & 0.69 & 2.63 & 0.10 & -0.36 \\
\hline BLLX & 贵州龙里 Longli, Guizhou & $106.90^{\circ} \mathrm{E}, 26.43^{\circ} \mathrm{N}$ & 1,150 & 10 & 18 & 0.45 & 0.61 & 2.30 & 0 & -0.37 \\
\hline BLP & 云南兰坪 Lanping, Yunnan & $99.42^{\circ} \mathrm{E}, 26.46^{\circ} \mathrm{N}$ & 2,540 & 12 & 24 & 0.61 & 0.98 & 2.93 & 0 & -0.57 \\
\hline BMG & 云南马关 Maguan, Yunnan & $104.39^{\circ} \mathrm{E}, 23.02^{\circ} \mathrm{N}$ & 1,340 & 12 & 24 & 0.53 & 0.79 & 2.80 & 0.02 & -0.45 \\
\hline BML & 四川木里 Muli, Sichuan & $101.25^{\circ} \mathrm{E}, 27.97^{\circ} \mathrm{N}$ & 2,500 & 12 & 26 & 0.61 & 0.96 & 3.05 & 0.19 & -0.55 \\
\hline $\mathrm{BNH}$ & 云南南华 Nanhua, Yunnan & $101.01^{\circ} \mathrm{E}, 25.33^{\circ} \mathrm{N}$ & 2,320 & 9 & 21 & 0.52 & 0.75 & 2.73 & 0 & -0.39 \\
\hline BQJ & 云南曲靖 Qujing, Yunnan & $103.91^{\circ} \mathrm{E}, 25.36^{\circ} \mathrm{N}$ & 1,900 & 8 & 20 & 0.49 & 0.74 & - & - & -0.44 \\
\hline BQL & 贵州贵阳 Guiyang, Guizhou & $106.69^{\circ} \mathrm{E}, 26.60^{\circ} \mathrm{N}$ & 1,160 & 6 & 17 & 0.45 & 0.60 & - & - & -0.24 \\
\hline BSZ & 云南师宗 Shizong, Yunnan & $103.98^{\circ} \mathrm{E}, 24.82^{\circ} \mathrm{N}$ & 1,870 & 12 & 25 & 0.52 & 0.71 & 2.91 & 0.03 & -0.34 \\
\hline BTM & 西藏林芝 Linzhi, Xizang & $96.58^{\circ} \mathrm{E}, 30.50^{\circ} \mathrm{N}$ & 4,720 & 15 & 25 & 0.59 & 0.85 & 2.83 & 0.09 & -0.41 \\
\hline BWM & 云南禄劝 Luquan, Yunnan & $102.83^{\circ} \mathrm{E}, 26.02^{\circ} \mathrm{N}$ & 2,760 & 14 & 22 & 0.47 & 0.69 & 2.49 & 0 & -0.46 \\
\hline BWN & 贵州威宁 Weining, Guizhou & $104.11^{\circ} \mathrm{E}, 26.74^{\circ} \mathrm{N}$ & 2,450 & 13 & 24 & 0.48 & 0.68 & 2.61 & 0 & -0.38 \\
\hline BWX & 云南维西 Weixi, Yunnan & $99.02^{\circ} \mathrm{E}, 27.80^{\circ} \mathrm{N}$ & 2,290 & 9 & 24 & 0.59 & 0.87 & 2.97 & 0.05 & -0.43 \\
\hline BXC & 四川西昌 Xichang, Sichuan & $102.35^{\circ} \mathrm{E}, 27.70^{\circ} \mathrm{N}$ & 2,680 & 11 & 20 & 0.39 & 0.57 & 2.38 & 0.03 & -0.41 \\
\hline $\mathrm{BXCX}$ & 云南西畴 Xichou, Yunnan & $104.79^{\circ} \mathrm{E}, 23.38^{\circ} \mathrm{N}$ & 1,450 & 9 & 25 & 0.53 & 0.75 & 2.86 & 0 & -0.37 \\
\hline BXJ & $\begin{array}{l}\text { 云南文山新街 Xinjie, Wenshan, } \\
\text { Yunnan }\end{array}$ & $104.00^{\circ} \mathrm{E}, 23.20^{\circ} \mathrm{N}$ & 1,880 & 15 & 23 & 0.53 & 0.81 & 2.76 & 0 & -0.49 \\
\hline BXZC & 云南开远 Kaiyuan, Yunnan & $103.62^{\circ} \mathrm{E}, 23.79^{\circ} \mathrm{N}$ & 1,480 & 10 & 21 & 0.54 & 0.80 & 2.77 & 0 & -0.43 \\
\hline BYG & 西藏波密 Bomi, Xizang & $95.57^{\circ} \mathrm{E}, 30.50^{\circ} \mathrm{N}$ & 5,230 & 15 & 22 & 0.58 & 0.88 & 2.79 & 0.01 & -0.48 \\
\hline BYXB & 四川越西 Yuexi, Sichuan & $102.45^{\circ} \mathrm{E}, 28.76^{\circ} \mathrm{N}$ & 2,970 & 12 & 16 & 0.39 & 0.60 & 2.12 & 0 & -0.49 \\
\hline BZNX & 云南文山 Wenshan, Yunnan & $104.42^{\circ} \mathrm{E}, 23.69^{\circ} \mathrm{N}$ & 1,570 & 15 & 25 & 0.55 & 0.81 & 2.97 & 0 & -0.45 \\
\hline
\end{tabular}

$A_{\mathrm{O}}$ : 等位基因数量; $H_{\mathrm{E}}$ : 期望杂合度; $H_{\mathrm{O}}$ ：观测杂合度; $R_{\mathrm{S}}$ : 等位基因丰富度; $P_{\mathrm{AR}}$ ：私有等位基因丰富度; $F_{\mathrm{IS}}$ ：近交系数(粗体标出的种群表示 符合哈温平衡)。

$A_{\mathrm{O}}$, Allele number; $H_{\mathrm{E}}$, Expected heterozygosity; $H_{\mathrm{O}}$, Observed heterozygosity; $R_{\mathrm{S}}$, Allelic richness; $P_{\mathrm{AR}}$, Private allelic richness; $F_{\mathrm{IS}}$, Inbreeding coefficient. Population in bold indicates it is under Hardy-Weinberg equilibrium.

物上添加苂光标记后(附录1), 所有个体的PCR产物 送到上海生工生物工程技术服务有限公司进行分 型分析。

\section{3 遗传多样性和遗传结构分析}

通过GENEMAPPER 3.7 (Applied Biosystems) 确定微卫星等位基因的大小。通过Micro-checker 2.2 (van Oosterhout et al, 2004)检测其是否存在无效等 位基因(null alleles)。对于不同微卫星位点, 使用
FSTAT 2.9.3 (Goudet, 2001) 计算等位基因数量 $\left(A_{\mathrm{O}}\right)$ 、观察杂合度 $\left(H_{\mathrm{O}}\right)$ 、总体遗传多样性 $\left(H_{\mathrm{T}}\right)$ 、遗传 多样性 $\left(H_{\mathrm{S}}\right)$ 和遗传分化系数 $\left(F_{\mathrm{ST}}\right)$ 。对于不同种群, 在GenAlEx 6.5 (Peakall \& Smouse, 2012)中计算等 位基因数量、观察杂合度、期望杂合度 $\left(H_{\mathrm{E}}\right)$ 和种群 间遗传分化; 在hp-rare 1.0 (Kalinowski, 2005)中计 算等位基因丰富度 $\left(R_{\mathrm{S}}\right)$ 和私有等位基因丰富度 $\left(P_{\mathrm{AR}}\right)$ 。通过FSTAT 2.9.3检测所有位点两两间是否 
存在连锁不平衡并通过经Bonferroni校正的近交系 数(inbreeding coefficient; $F_{\mathrm{IS}}$ )判断所有种群是否符 合Hardy-Weinberg平衡。

在基于贝叶斯聚类分析的STRUCTURE 2.3.3 (Falush et al, 2007)中推测扁核木群体间遗传变异的 地理分布模式。选择混合祖先模型 (amdmixture model)和关联等位基因频率模型 (correlated allele frequency model)。设置 burn-in 长度为 $1 \times 10^{5}$; MCMC长度为 $1 \times 10^{6}$ 。设置 $K$ 值为 $1-20$, 每个 $K$ 值重 复10次运算。通过HARVST (http://taylor0.biology. ucla.edu/structureHarvester/) 计算后验概率 $[\ln \mathrm{P}(D)]$ 和后验概率的变化速率 $(\Delta K)$, 并依此推测可能性最 大的分组(Pritchard et al, 2000; Evanno et al, 2005)。 进一步, 我们通过不同种群间遗传分化的主成分分 析推测不同遗传群组间是否有显著分化。对于不同 遗传群组, 等位基因数量、观察杂合度和期望杂合 度以及等位基因丰富度和私有等位基因丰富度分 别由GenAlEx 6.5和hp-rare 1.0计算。不同遗传群组 间的遗传分化由GenAlEx 6.5计算。

\section{4 遗传分化成因}

为探究扁核木种群遗传分化的成因, 我们对遗 传距离与地理距离和环境差异进行了相关性分析。 首先, 将GenAlEx 6.5计算的不同种群间遗传分化 作为遗传距离。其次, 我们下载了WorldClim数据库 (Hijmans et al, 2005)中指示温度和降水的19个气候 变量, 在ArcGiS 10.2 (ESRI, Redlands, CA, USA)中 通过Spatial Analyst Tools功能提取不同种群采样点 的气候变量。在R 3.5 .3 (https://www.r-project.org/) 中计算不同种群气候间的欧式距离, 作为两两种群 间的环境差异。同时，在R中通过“princomp”功能对 气候变量进行主成分分析以推测不同群组间的气 候是否存在显著差异。然后, 通过GenAlEx 6.5中经 过对数转换的地理距离 $[\ln (1+$ GGD) ]得到地理距 离矩阵。最后, 在 R中使用 “partial.mantel.test”功能 进行Mantel test, 即遗传距离与地理距离或环境差 异的相关性, 以及partial Mantel test, 即遗传距离与 地理距离(去除环境差异影响)或遗传距离与环境差 异(去除地理距离影响)的相关性, 并基于 $1 \times 10^{4}$ 个 置换计算显著性。

\section{5 种群历史}

在DIYABC 2.0 (Cornuet et al, 2014)中, 我们通 过模拟探究扁核木不同遗传群组间可能的分化历
史，并对有效种群大小和分化时间等参数进行估 计。首先, 喜马拉雅群组与其他群组存在明显的遗 传分化, 我们将喜马拉雅群组最先分化作为不同模 型设置的前提。其次, 由于核微卫星位点数较少且 需要估测的参数较多, 我们对模型的设置进行了简 化。仅比较了 3 种不同分化历史的可能性, 3 个模型 不同之处在于除喜马拉雅群组外的其余 3 个群组中 哪个群组先分化。4 个群组的有效种群大小设置为 $10-10,000$, 分化时间设置为 $10-10,000$, 其中 $t 1<t 2$, $t 2<t 3$ 。突变速率设置为 $1 \times 10^{-6}$ 至 $1 \times 10^{-4}$, mean coefficient $P$ 设置为 $0-1$ 。所有参数的分布为均匀分 布。

在3个模型的模拟中, 群组内统计了等位基因 数目均值(mean number of alleles)和基因多样性均 值(mean genic diversity)指标, 群组间我们统计遗传 分化 $\left(F_{\mathrm{ST}}\right) 、$ mean index of classification和shared allele distance指标。每个模型运行 $1 \times 10^{6}$ 次模拟后，基于 $1 \%\left(1 \times 10^{4}\right)$ 最接近观测值的数据通过逻辑回归确 定最优模型后估测种群分化历史中的各项参数。

\section{结果}

\section{1 遗传多样性}

我们篎选的7对扁核木核微卫星位点均未发现 无效等位基因(附录1)且不存在连锁位点。对于不同 种群, 等位基因数量为15-27个, 期望杂合度和观 测杂合度的范围分别是 $0.38-0.64$ 和 $0.57-0.98$, 等位 基因丰富度和私有等位基因丰富度最低分别是 2.12 和 0 , 最高分别是3.28和 0.19 (表1)。基于近交系数, 我们发现除BQL (贵州贵阳)种群外, 其余种群均不 符合Hardy-Weinberg平衡(表1)，可能与非随机交配 或种群内存在亚结构等有关。对于不同位点, 等位 基因数量为5-9个, 平均值为6.57。Pru_17717位点的 观测杂合度、遗传多样性和总体遗传多样性均为最 低，其余位点的遗传多样性水平类似。Pru_17717的 遗传分化水平最高, 其余位点的较低, 平均水平为 0.21 (附录2)。

\section{2 遗传结构}

在STRUCTURE分析中，根据 $\ln \mathrm{P}(D)$ 和 $\Delta K$ 的分 布(附录3), 我们推测扁核木 32 个种群可能的遗传 分组为 $K=2$ 或 $K=4$ 。当 $K=2$ 时, 喜马拉雅地区的 4 个种群(BJL、BBM、BYG和BTM)与其余种群分为 
具有显著遗传分化的 2 个遗传群组, 群组间几乎没 有基因交流。当 $K=4$ 时, 喜马拉雅群组保留, 其余 种群划分为 3 个不同的遗传群组, 自西向东将其编 码为横断山、云贵高原西部和云贵高原东部群组(图 1)。喜马拉雅和云贵高原东部群组是包含其他群组 遗传成分较少的群组, 而横断山和云贵高原西部群 组包含了较多其他群组的遗传成分, 尤其是云贵高 原西部群组有大量云贵高原东部群组的遗传组分 (图1)。
种群间遗传分化的主成分分析支持了 4 个遗传 群组的划分, 喜马拉雅和云贵高原东部群组与其他 2 个群组的差异更明显(附录4)。其中遗传分化水平 最高的为喜马拉雅和云贵高原东部群组间 $\left(F_{\mathrm{ST}}=\right.$ $0.16)$, 其次为喜马拉雅和云贵高原西部 $\left(F_{\mathrm{ST}}=\right.$ $0.15)$ 、喜马拉雅和横断山 $\left(F_{\mathrm{ST}}=0.11\right)$ 、云贵高原东 西部 $\left(F_{\mathrm{ST}}=0.11\right)$ 以及横断山和云贵高原东部 $\left(F_{\mathrm{ST}}=\right.$ 0.09)群组之间, 最低的为横断山和云贵高原西部群 组间 $\left(F_{\mathrm{ST}}=0.06\right) 。 4$ 个群组有相似的等位基因数量、

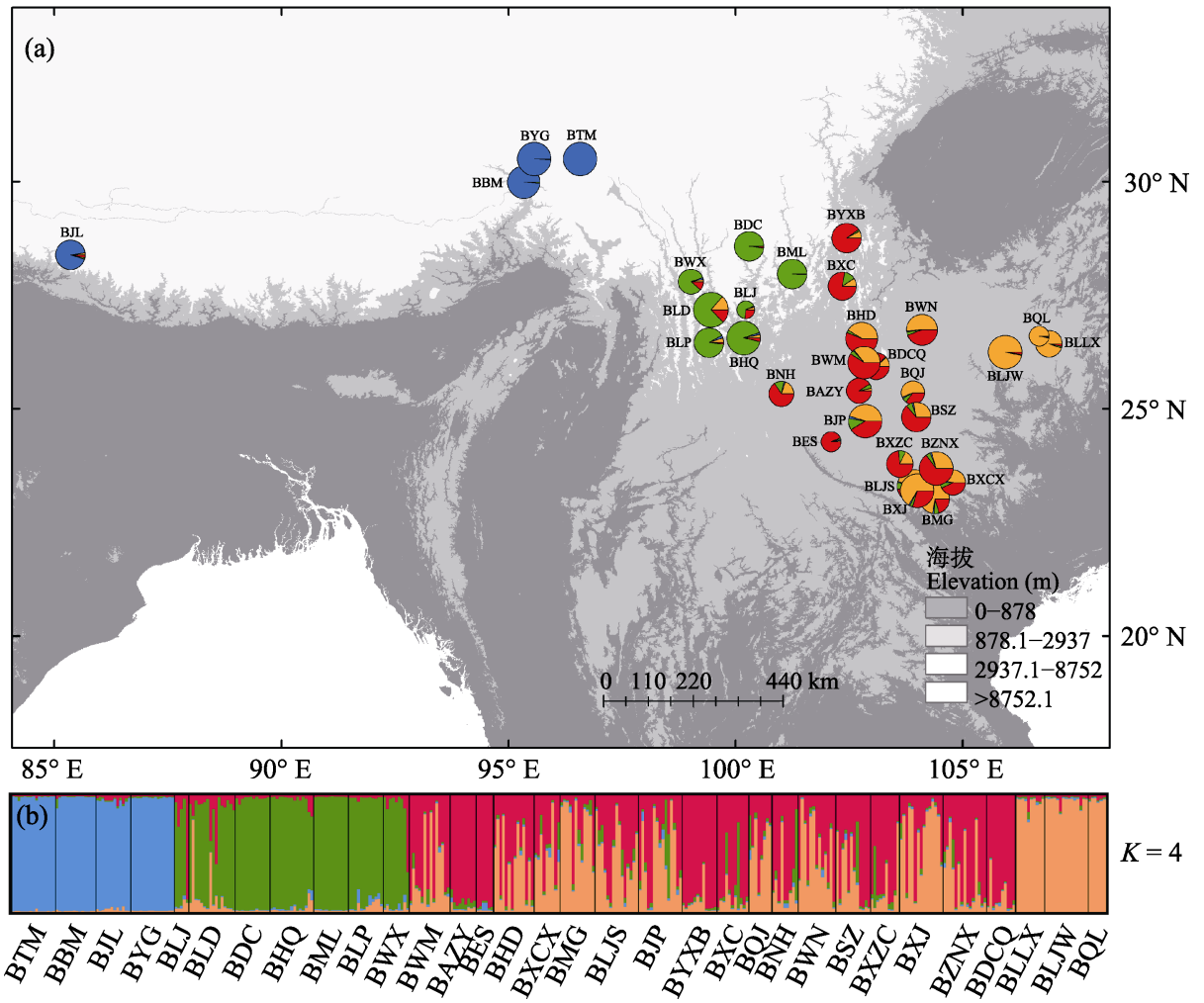

图1 扁核木 32 个种群遗传群组 $(K=4)$ 的分布 $(a)$ 和不同个体所属群组比值的柱状图(b)。蓝色为喜马拉雅群组, 绿色为横断山 群组, 红色和橙色分别是云贵高原西部和东部群组。种群代号见表1。

Fig. 1 Color-coded clustering (a) and histogram of the cluster assignment of different individuals (b) of the 32 Prinsepia utilis populations when $K=4$. Blue represents Himalayas group, green represents Hengduan Mountains group, red and orange represent west and east Yunnan-Guizhou Plateau group, respectively. Population codes are the same as that in Table 1.

表2 扁核木不同遗传群组的遗传多样性

Table 2 Genetic diversity of different genetic cluster of Prinsepia utilis

\begin{tabular}{llllll}
\hline 遗传群组 & 等位基因数量 & $\begin{array}{l}\text { 观测杂合度 } \\
\text { Genetic cluster }\end{array}$ & $A_{\mathrm{O}}$ & $H_{\mathrm{O}}$ & \multicolumn{2}{l}{$\begin{array}{l}\text { 期望杂合度 } \\
H_{\mathrm{E}}\end{array}$} & \multicolumn{2}{l}{$\begin{array}{l}\text { 等位基因丰富度 } \\
R_{\mathrm{S}}\end{array}$} & $\begin{array}{l}\text { 私有等位基因丰富度 } \\
P_{\mathrm{AR}}\end{array}$ \\
\hline 喜马拉雅 Himalayas & 30 & 0.79 & 0.62 & 3.18 & 0.70 \\
横断山 Hengduan Mountains & 35 & 0.86 & 0.65 & 3.39 & 0.53 \\
云贵高原西部 West Yunnan-Guizhou Plateau & 36 & 0.73 & 0.56 & 3.04 & 0.19 \\
云贵高原东部 East Yunnan-Guizhou Plateau & 26 & 0.65 & 0.50 & 2.69 & 0.24 \\
\hline
\end{tabular}

$A_{\mathrm{O}}$, Allele number; $H_{\mathrm{O}}$, Observed heterozygosity; $H_{\mathrm{E}}$, Expected heterozygosity; $R_{\mathrm{S}}$, Allelic richness; $P_{\mathrm{AR}}$, Private allelic richness. 
观测杂合度、期望杂合度和等位基因丰富度, 喜马 拉雅群组的私有等位基因丰富度最高 $\left(P_{\mathrm{AR}}=0.70\right)$ (表2)。

\section{3 遗传分化成因}

在Mantel test中, 遗传距离随着地理距离 $(r=$ $0.12, P=0.04)$ 和环境差异 $(r=0.48, P=0)$ 的增大而 显著升高。在去除环境差异影响后, partial Mantel test显示遗传距离与地理距离间无显著相关 $(r=$ $0.08, P=0.10)$; 而在去除地理距离影响后, 遗传距 离与环境差异间仍存在显著相关性 $(r=0.47, P=$ $0)$ 。在不同种群气候变量的主成分分析中, 前 2 个主 成分可以解释 $84.7 \%$ 的种群间气候差异。在散点图 中, 不同群组间的气候存在不同程度的差异, 其中 喜马拉雅和云贵高原东部群组与其他 2 个群组间的 差异最明显, 横断山和云贵高原东西部群组间的气 候存在一定的重叠(图2)。

\section{4 种群分化历史}

在DIYABC分析中, 横断山和云贵高原西部群 组在云贵高原东部群组形成后再形成的情景得到 了最高的支持 $(\mathrm{PP}=0.57)$, 其他 2 种模型的后验概率 分别是 0.25 和 0.18 (图3)。在最优情景中, 扁核木 4 个群组有效种群大小类似, 为5.8-6.8 $\times 10^{4}$ 。不同遗 传群组依次分化的 $t 1-3$ 分别为 $8.53 \times 10^{3} 、 1.25 \times 10^{4}$ 和 $5.11 \times 10^{4}$ 世代，基于扁核木的世代时间为 10 年左 右(和琼姬等, 2016), 不同的分化时间 T1-3分别为 $8.53 \times 10^{4} 、 1.25 \times 10^{5}$ 和 $5.11 \times 10^{5}$ 年。扁核木核微卫 星每世代的突变速率为4.30 $\times 10^{-6}(95 \%$ 置信区间: $2.19 \times 10^{-6}-1.01 \times 10^{-5}$ ) (表3，附录5)。

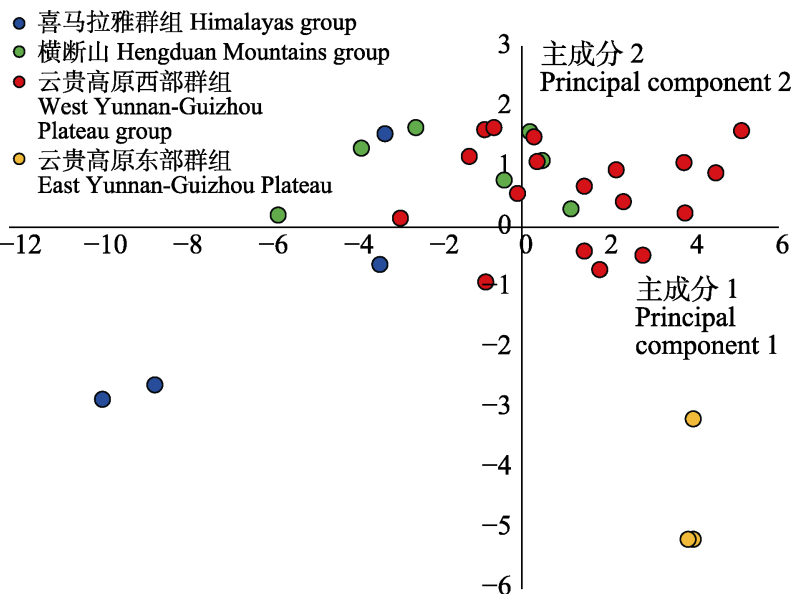

图2 扁核木32个种群间气候变量的主成分分析, 其中不同 遗传群组的颜色与图1中相对应。

Fig. 2 Principal component analysis of climate variables of 32 Prinsepia utilis populations. Coded colors of different genetic clusters correspond to those in Fig. 1.

\section{3 讨论}

\section{1 扁核木遗传结构及其成因}

通过多位点的核微卫星信息，我们发现扁核木 种群存在显著的遗传结构。首先，遗传结构最显著 的存在于喜马拉雅地区与横断山和云贵高原地区 种群间(图1), 形成了最高水平的群组间遗传分化。 扁核木属生物地理研究也显示扁核木种群有类似 格局，在叶绿体和核糖体ITS基因中，虽然不同的 遗传谱系没有明显的地理结构，但喜马拉雅地区种 群有独特的单倍型(Ma et al, 2019; Jin et al, 2020)。 该遗传分化可能由南北走向的MSD (Ward，1921; 叶俊伟等, 2017)造成。STRUCTURE和种群间遗传
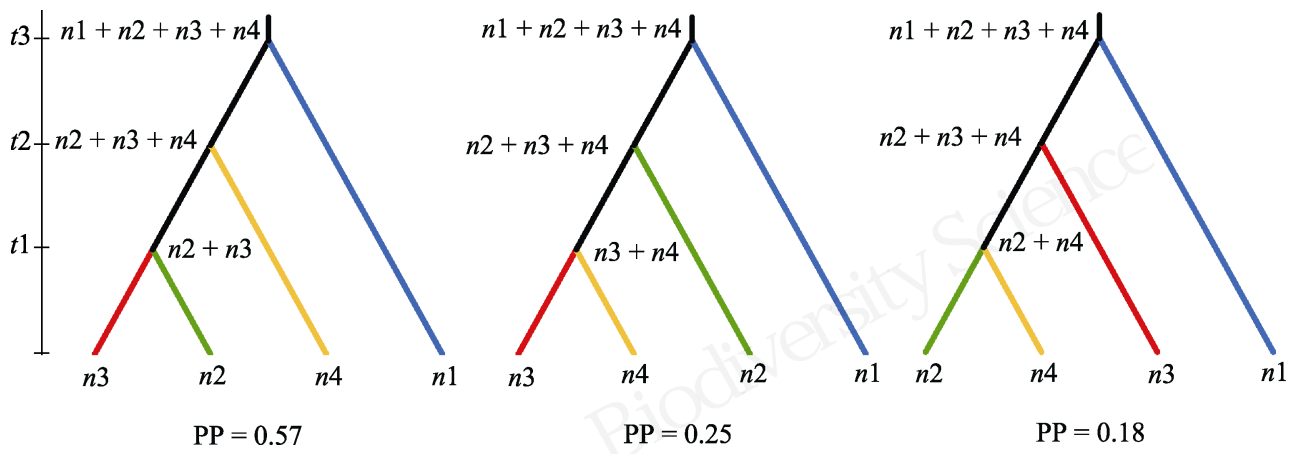

图3 DIYABC分析中3种不同的扁核木种群分化历史示意图。n1-4分别为喜马拉雅、横断山以及云贵高原西部和东部遗传群 组的有效种群大小, $t$ - 3 分别为不同分化事件的分化时间(以世代为单位), PP为后验概率。

Fig. 3 Illustration of three different scenarios of divergence history of Prinsepia utilis populations. n1-4 represent effective population sizes of Himalayas, Hengduan Mountains, west and east Yunnan-Guizhou Plateau genetic groups, respectively; $t 1-3$ represent divergence time of different divergence events (in generations); and PP represents posterior probability. 
叶俊伟和田斌: 中国西南地区重要木本油料植物扁核木的遗传结构及成因 1635

表3 DIYABC模拟扁核木种群最优分化历史中不同参数的后验中位值及 $95 \%$ 置信区间

Table 3 Posterior median and 95\% highest posterior density interval (HPD) estimate for demographic parameters in the most possible divergence scenario of Prinsepia utilis in DIYABC

\begin{tabular}{|c|c|c|c|c|c|c|c|c|c|}
\hline & $n 1$ & $n 2$ & $n 3$ & $n 4$ & $T 1$ & $T 2$ & $T 3$ & $\mu$ & $P$ \\
\hline 中位值 Median & $6.84 \times 10^{4}$ & $6.30 \times 10^{4}$ & $5.84 \times 10^{4}$ & $3.90 \times 10^{4}$ & $8.53 \times 10^{4}$ & $1.25 \times 10^{5}$ & $5.11 \times 10^{5}$ & $4.30 \times 10^{-6}$ & 0.21 \\
\hline $\begin{array}{l}\text { 95\%置信区间下限 } \\
\text { 95\% HPD lower }\end{array}$ & $3.30 \times 10^{4}$ & $2.68 \times 10^{4}$ & $2.39 \times 10^{4}$ & $1.21 \times 10^{4}$ & $1.80 \times 10^{4}$ & $2.73 \times 10^{4}$ & $1.92 \times 10^{5}$ & $2.19 \times 10^{-6}$ & 0.11 \\
\hline $\begin{array}{l}\text { 95\%置信区间上限 } \\
\text { 95\% HPD upper }\end{array}$ & $9.53 \times 10^{4}$ & $9.44 \times 10^{4}$ & $9.15 \times 10^{4}$ & $8.50 \times 10^{4}$ & $3.49 \times 10^{5}$ & $4.49 \times 10^{5}$ & $8.82 \times 10^{5}$ & $1.01 \times 10^{-5}$ & 0.70 \\
\hline
\end{tabular}

n1-4: 喜马拉雅、横断山、云贵高原西部和东部遗传群组的有效种群大小; $T 1-3:$ 分化时间(以年为单位); $\mu$ : 突变速率(每位点每世代); $P$ : 广 义逐步替代模型中多步突变的比例。

n1-4, Effective population sizes of Himalayas, Hengduan Mountains, west and east Yunnan-Guizhou Plateau genetic clusters, respectively; T1-3, Divergence time (in years); $\mu$, Mutation rate (per generation per locus); $P$, The proportion of multiple step mutations in the generalized stepwise model.

分化的主成分分析均显示除喜马拉雅地区外的种 群可划分为3个遗传群组(图1, 附录4)。3个群组均 大致呈南北向的分布格局, 与叶绿体单倍型的格局 类似(Ma et al, 2019)。推测南北向的高山峡谷地形 有利于群组内南北种群的基因交流(Sun et al, 2017; Muellner-Riehl, 2019)。

遗传成因分析表明扁核木种群间显著的遗传 分化主要由环境差异导致。虽然以往研究表明地理 隔离在种群分化中至关重要(刘杰等, 2017), 但其 对扁核木种群遗传分化的作用较小。可能是因为: (1) 虽然扁核木的花粉由小型昆虫传播, 但其种子可由 鸟类传播, 长距离传播产生的基因流会导致不同群 组间的遗传成分混杂, 尤其是横断山和云贵高原西 部群组间(图1)。(2)种群分化历史显示扁核木分布区 东西两侧种群先分化后, 分布区中间的 2 个群组才 形成(图2), 因此在去除环境差异的影响后, 遗传距 离与地理距离没有显著相关性。4 个群组间的气候 存在不同程度的差异, 但气候存在显著差异的喜马 拉雅和云贵高原东部种群是否形成了适应性分化 (Liu et al, 2013), 仍需要进一步的探究。虽然喜马拉 雅地区西侧的BJL种群与横断山及云贵高原西部种 群的气候无明显差异, 但长距离和MSD的存在阻 碍了基因交流。

在扁核木属的生物地理学研究中, 扁核木种群 不同单倍型的共祖时间为中新世早中期(Ma et al, 2019; Jin et al, 2020), 而本研究中估测的不同群组 间分化时间均为晚更新世(表3), 是由于核微卫星 的突变速率远小于叶绿体/核糖体的突变速率导致 (白伟宁和张大勇, 2014)。因此, 虽然扁核木的进化 历史可追溯至中新世早期(Ma et al, 2019), 但其核 基因显著遗传结构的形成可能是由更新世冰期间
冰期交替的气候变化导致的。

\section{2 扁核木遗传资源评估}

扁核木为中国西南地区温带森林重要的木本 油料植物, 其遗传资源的评估对发展有效的保护和 利用至关重要(Li et al, 2019)。扁核木不仅在属内有 最高的遗传多样性(Ma et al, 2019), 种群内的遗传 多样性同样较高(表1), 且有明显的遗传结构(图1, 2)，拥有丰富的遗传资源。

首先, 喜马拉雅种群是拥有最高水平私有等位 基因频率的独特遗传群组(表2), 与其他群组间存 在显著的气候差异(BJL种群除外), 存在发生适应 性分化的可能(Liu et al, 2013)。在基于最大熵模型 MaxEnt对扁核木过去(末次盛冰期)、现在和未来 (2050年)潜在分布区的模拟中(Ma et al, 2019), 相比 于现在, 过去和未来喜马拉雅地区生境的适合度更 低。因此, 喜马拉雅地区是需要我们首要关注的区 域。其次, 云贵高原东部群组也几乎不包含其他群 组遗传成分(图1)。该区域的环境尤其是温度与其他 区域显著不同(图2), 可能会导致扁核木出油率和 有效成分含量等的不同。在潜在分布区的模拟中， 四川盆地西侧和云贵高原地区是过去和未来扁核 木的最适分布区(Ma et al, 2019)。横断山和云贵高 原西部群组混合了大量其他群组的遗传成分, 而在 生态位模拟中，未来横断山地区扁核木生存的适合 度将有所降低(Ma et al, 2019), 预示着种群有破碎 化或收缩的趋势。综上, 我们首先需要重点保护喜 马拉雅和云贵高原地区的种群, 尤其是云贵高原东 部群组所在区域; 对横断山地区野外种群的保护和 种质的收集也需要高度重视。下一步, 我们将根据 遗传资源评估的结果, 对不同区域扁核木种群的结 实率、出油率和有效成分含量以及所在区域温度、 


\section{土壤和降雨量等因子进行更为详尽的调查。}

致谢：感谢刘家奇在样品采集、分子实验和数据处 理中提供的帮助。

\section{ORCID}

叶俊伟 (iD https://orcid.org/0000-0002-3614-3631

田斌 (iD https://orcid.org/0000-0003-2325-724X

\section{参考文献}

Bai WN, Zhang DY (2014) Current status and future directions in plant phylogeography. Chinese Bulletin of Life Sciences, 26, 125-137. (in Chinese with English abstract) [白伟宁, 张大勇 (2014) 植物亲缘地理学的研究现状与发展趋势. 生命科学, 26, 125-137.]

Cornuet JM, Pudlo P, Veyssier J, Dehne-Garcia A, Gautier M, Leblois R, Marin JM, Estoup A (2014) DIYABC v2.0: A software to make approximate Bayesian computation inferences about population history using single nucleotide polymorphism, DNA sequence and microsatellite data. Bioinformatics, 30, 1187-1189.

Ding WN, Ree RH, Spicer RA, Xing YW (2020) Ancient orogenic and monsoon-driven assembly of the world's richest temperate alpine flora. Science, 369, 578-581.

Doyle JJ, Doyle JL (1990) Isolation of Plant DNA from fresh tissue. Focus, 12, 13-15.

Evanno G, Regnaut S, Goudet J (2005) Detecting the number of clusters of individuals using the software STRUCTURE: A simulation study. Molecular Ecology, 14, 2611-2620.

Falush D, Stephens M, Pritchard JK (2007) Inference of population structure using multilocus genotype data: Dominant markers and null alleles. Molecular Ecology Notes, 7, 574-578.

Goudet J (2001) FSTAT, A Program to Estimate and Test Gene Diversities and Fixation Indices (version 2.9.3). https://www2.unil.ch/popgen/softwares/fstat.htm. (accessed on 2021-08-30)

Guan B, Peng CC, Wang CH, Jin HZ, Zhang WD (2014) Chemical constituents from the aerial parts of Prinsepia utilis. Chemistry of Natural Compounds, 50, 1106-1107.

Guan B, Peng CC, Zeng Q, Cheng XR, Yan SK, Jin HZ, Zhang WD (2013) Cytotoxic pentacyclic triterpenoids from Prinsepia utilis. Planta Medica, 79, 365-368.

He QJ, He JW, Wang YP, Mu YQ, Yang WH, Yang HT, Huang XE, Wu YB, He SL, He JP (2016) Overview of Prinsepia utilis Royle. Chinese Agricultural Science Bulletin, 32(7), 74-78. (in Chinese with English abstract) [和琼姬, 和加卫, 王宇萍, 木永青, 杨文宏, 杨洪涛, 黄杏娥, 吴 永斌, 和寿莲, 和建平 (2016) 青刺果研究概述. 中国农 学通报, 32(7), 74-78.]

Hijmans RJ, Cameron SE, Parra JL, Jones PG, Jarvis A (2005)
Very high resolution interpolated climate surfaces for global land areas. International Journal of Climatology, 25, 19651978.

Huang SQ, Ma YL, Zhang CT, Cai SB, Pang MJ (2017) Bioaccessibility and antioxidant activity of phenolics in native and fermented Prinsepia utilis Royle seed during a simulated gastrointestinal digestion in vitro. Journal of Functional Foods, 37, 354-362.

Jin WY, Li HW, Wei R, Huang BH, Liu B, Sun TT, Mabberley DJ, Liao PC, Yang Y (2020) New insights into biogeographical disjunctions between Taiwan and the Eastern Himalayas: The case of Prinsepia (Rosaceae). Taxon, 69, 278-289.

Ju MM, Ma HC, Xin PY, Zhou ZL, Tian B (2015) Development and characterization of EST-SSR markers in Bombax ceiba (Malvaceae). Applications in Plant Sciences, 3, 1500001.

Kalinowski ST (2005) Hp-rare 1.0: A computer program for performing rarefaction on measures of allelic richness. Molecular Ecology Notes, 5, 187-189.

Li Q, Zhao Y, Xiang XG, Chen JK, Rong J (2019) Genetic diversity of crop wild relatives under threat in Yangtze River basin: Call for enhanced in situ conservation and utilization. Molecular Plant, 12, 1535-1538.

Li XW, Li J (1993) A preliminary floristic study on the seed plants from the region of Hengduan Mountain. Acta Botanica Yunnanica, 15, 217-231. (in Chinese with English abstract) [李锡文, 李捷 (1993) 横断山脉地区种子植物 区系的初步研究. 云南植物研究, 15, 217-231.]

Liu J, Luo YH, Li DZ, Gao LM (2017) Evolution and maintenance mechanisms of plant diversity in the QinghaiTibet Plateau and adjacent regions: Restrospect and prospect. Biodiversity Science, 25, 163-174. (in Chinese with English abstract) [刘杰, 罗亚皇, 李德铢, 高连明 (2017) 青藏高原及毗邻区植物多样性演化与维持机制: 进展及展望. 生物多样性, 25, 163-174.]

Liu J, Möller M, Provan J, Gao LM, Poudel RC, Li DZ (2013) Geological and ecological factors drive cryptic speciation of yews in a biodiversity hotspot. New Phytologist, 199, 1093-1108.

Lu LM, Mao LF, Yang T, Ye JF, Liu B, Li HL, Sun M, Miller JT, Mathews S, Hu HH, Niu YT, Peng DX, Chen YH, Smith SA, Chen M, Xiang KL, Le CT, Dang VC, Lu AM, Soltis PS, Soltis DE, Li JH, Chen ZD (2018) Evolutionary history of the angiosperm flora of China. Nature, 554, 234-238.

Luo D, Yue JP, Sun WG, Xu B, Li ZM, Comes HP, Sun H (2016) Evolutionary history of the subnival flora of the Himalaya-Hengduan Mountains: First insights from comparative phylogeography of four perennial herbs. Journal of Biogeography, 43, 31-43.

Ma XG, Wang ZW, Tian B, Sun H (2019) Phylogeographic analyses of the East Asian endemic genus Prinsepia and the 
role of the East Asian monsoon system in shaping a north-south divergence pattern in China. Frontiers in Genetics, 10, 128.

Ma YZ, Wang J, Hu QJ, Li JL, Sun YS, Zhang L, Abbott RJ, Liu JQ, Mao KS (2019) Ancient introgression drives adaptation to cooler and drier mountain habitats in a cypress species complex. Communications Biology, 2, 213.

Muellner-Riehl AN (2019) Mountains as evolutionary arenas: Patterns, emerging approaches, paradigm shifts, and their implications for plant phylogeographic research in the Tibeto-Himalayan region. Frontiers in Plant Science, 10, 195.

Peakall R, Smouse PE (2012) GenAlEx 6.5: Genetic analysis in Excel. Population genetic software for teaching and research-An update. Bioinformatics, 28, 2537-2539.

Pritchard JK, Stephens M, Donnelly P (2000) Inference of population structure using multilocus genotype data. Genetics, 155, 945-959.

Sun H, Zhang JW, Deng T, Boufford DE (2017) Origins and evolution of plant diversity in the Hengduan Mountains, China. Plant Diversity, 39, 161-166.

van Oosterhout C, Hutchinson WF, Wills DPM, Shipley P
(2004) Micro-checker: Software for identifying and correcting genotyping errors in microsatellite data. Molecular Ecology Notes, 4, 535-538.

Wang S, Shi C, Gao LZ (2013) Plastid genome sequence of a wild woody oil species, Prinsepia utilis, provides insights into evolutionary and mutational patterns of Rosaceae chloroplast genomes. PLoS ONE, 8, e73946.

Ward FK (1921) The Mekong-Salween Divide as a geographical barrier. The Geographical Journal, 58, 49-56.

Xing YW, Ree RH (2017) Uplift-driven diversification in the Hengduan Mountains, a temperate biodiversity hotspot. Proceedings of the National Academy of Sciences, USA, 114, E3444-E3451.

Ye JW, Zhang Y, Wang XJ (2017) Phylogeographic breaks and the mechanisms of their formation in the Sino-Japanese floristic region. Chinese Journal of Plant Ecology, 41, 1003-1019. (in Chinese with English abstract) [叶俊伟, 张 阳, 王晓娟 (2017) 中国-日本植物区系中的谱系地理间 断及其形成机制. 植物生态学报, 41, 1003-1019.]

(责任编委：邱英雄 责任编辑：时意专)

\section{附录 Supplementary Material}

附录1 扁核木7个核微卫星位点的基本信息

Appendix 1 Information of seven nuclear microsatellite loci developed of Prinsepia utilis https://www.biodiversity-science.net/fileup/PDF/2021341-1.pdf

\section{附录2 扁核木7个核微卫星位点的遗传多样性和遗传分化}

Appendix 2 Measures of genetic diversity and genetic differentiation of seven nuclear microsatellite loci of Prinsepia utilis https://www.biodiversity-science.net/fileup/PDF/2021341-2.pdf

\section{附录3 扁核木32个种群STRUCTURE分析中后验概率 $[\ln P(D)](a)$ 和后验概率的变化速率 $(\Delta K)(b)$ 分布}

Appendix 3 Distribution of the change in log-likelihood of the data for each run, $\ln \mathrm{P}(D)(\mathrm{a})$, and $\Delta K$, the second order rate of change of $\ln \mathrm{P}(D)$ between successive $K$-values (b) in STRUCTURE analysis of 32 populations of Prinsepia utilis https://www.biodiversity-science.net/fileup/PDF/2021341-3.pdf

附录4 扁核木32个种群间遗传分化的主成分分析。其中不同遗传群组的颜色与图1中相对应。

Appendix 4 Principal component analysis of pairwised genetic differentiation of 32 Prinsepia utilis populations, coded colour of different genetic clusters are corresponded to that in Fig. 1

https://www.biodiversity-science.net/fileup/PDF/2021341-4.pdf

附录5 DIYABC分析最优种群分化历史不同参数的先验(红色线条)和后验分布(绿色线条)

Appendix 5 Prior (red line) and posterior (green line) distributions of different parameters of the most possible divergence scenario of Prinsepia utilis in DIYABC analysis https://www.biodiversity-science.net/fileup/PDF/2021341-5.pdf 
叶俊伟，田斌 (2021) 中国西南地区重要木本油料植物扁核木的遗传结构及成因. 生物多样性, 29, 16291637.

http://www.biodiversity-science.net/CN/10.17520/biods.2021341

\section{附录1 扁核木7个核微卫星位点的基本信息}

Appendix 1 Information of seven nuclear microsatellite loci developed of Prinsepia utilis

\begin{tabular}{|c|c|c|c|c|}
\hline 位点 Locus & 引物序列 Primer sequence $\left(5^{\prime}-3^{\prime}\right)$ & $\begin{array}{l}\text { 苂光标记 } \\
\text { Fluorescent label }\end{array}$ & $\begin{array}{l}\text { 重复模式 } \\
\text { Repeat motif }\end{array}$ & $\begin{array}{l}\text { 退火温度 } \\
\text { Annealing } \\
\text { temperature }\end{array}$ \\
\hline Pru_22139 & $\begin{array}{l}\text { 正向 Forward: } \\
\text { TCACTTCACCAAATACGCCA } \\
\text { 反向 Reversed: } \\
\text { AGGTTGAAAGCCCGGTAGTT }\end{array}$ & 6-FAM & $(\mathrm{TCT})_{7}$ & $56^{\circ} \mathrm{C}$ \\
\hline Pru_17717 & $\begin{array}{l}\text { 正向 Forward: } \\
\text { CTACTAGCCCAGTCCTCCCC } \\
\text { 反向 Reversed: } \\
\text { ACGAGGCAACAGAAAAAGGA }\end{array}$ & HEX & $(\mathrm{AG})_{9}$ & $56^{\circ} \mathrm{C}$ \\
\hline Pru_25578 & $\begin{array}{l}\text { 正向 Forward: } \\
\text { TGTACCGTTCGAATGAGGTG } \\
\text { 反向 Reversed: } \\
\text { CGGGTTGTGGGTTTAGTCAG }\end{array}$ & 6-FAM & $(\mathrm{AC})_{8}$ & $56^{\circ} \mathrm{C}$ \\
\hline Pru_25182 & $\begin{array}{l}\text { 正向 Forward: } \\
\text { CTTGGCTAAAGCAGCTCAGG } \\
\text { 反向 Reversed: } \\
\text { CCAAGTACCGCCAGAAGAAG }\end{array}$ & TAMRA & $(\mathrm{AGAA})_{6}$ & $56^{\circ} \mathrm{C}$ \\
\hline Pru_29944 & $\begin{array}{l}\text { 正向 Forward: } \\
\text { GGTTCCTCCCCCTATGAAGA } \\
\text { 反向 Reversed: } \\
\text { ACAATCTTCAGGCGCATTCT }\end{array}$ & HEX & $(\mathrm{GATCA})_{5}$ & $56^{\circ} \mathrm{C}$ \\
\hline Pru_28410 & $\begin{array}{l}\text { 正向 Forward: } \\
\text { TCCGTTCATCAATCACAATGA } \\
\text { 反向 Reversed: } \\
\text { ACAAGGCGTCAAGGTACGTC }\end{array}$ & 6-FAM & $(\mathrm{AG})_{10}$ & $54^{\circ} \mathrm{C}$ \\
\hline Pru_23037 & $\begin{array}{l}\text { 正向 Forward: } \\
\text { CGGGAAAACGTACCGACTAA } \\
\text { 反向 Reversed: } \\
\text { ATGGCTCTACATAATGCCCG }\end{array}$ & TAMRA & $(\text { TAGT })_{6}$ & $54^{\circ} \mathrm{C}$ \\
\hline
\end{tabular}


叶俊伟，田斌 (2021) 中国西南地区重要木本油料植物扁核木的遗传结构及成因. 生物多样性, 29, 16291637.

http://www.biodiversity-science.net/CN/10.17520/biods.2021341

\section{附录2 扁核木7个核微卫星位点的遗传多样性和遗传分化}

Appendix 2 Measures of genetic diversity and genetic differentiation of seven nuclear microsatellite loci of Prinsepia utilis

\begin{tabular}{llllll}
\hline 位点 Locus & $A_{\mathrm{O}}$ & $H_{\mathrm{O}}$ & $H_{\mathrm{S}}$ & $H_{\mathrm{T}}$ & $F_{\mathrm{ST}}$ \\
\hline Pru_22139 & 8 & 0.92 & 0.63 & 0.71 & 0.11 \\
Pru_17717 & 9 & 0.30 & 0.28 & 0.60 & 0.54 \\
Pru_25578 & 6 & 0.83 & 0.58 & 0.65 & 0.10 \\
Pru_25182 & 5 & 0.83 & 0.56 & 0.65 & 0.14 \\
Pru_29944 & 5 & 0.60 & 0.42 & 0.65 & 0.37 \\
Pru_28410 & 8 & 0.91 & 0.64 & 0.71 & 0.10 \\
Pru_23037 & 5 & 0.85 & 0.58 & 0.63 & 0.08 \\
平均值 Mean & 6.57 & 0.75 & 0.53 & 0.66 & 0.21 \\
\hline
\end{tabular}

$A_{\mathrm{O}}$ : 等位基因数量; $H_{\mathrm{O}}$ ：观测杂合度; $H_{\mathrm{S}}$ ：遗传多样性; $H_{\mathrm{T}}$ ：总体遗传多样性; $F_{\mathrm{ST}}$ ：遗传分化。 $A_{\mathrm{O}}$, Allele number; $H_{\mathrm{O}}$, Observed heterozygosity; $H_{\mathrm{S}}$, Gene diversity; $H_{\mathrm{T}}$, Overall gene diversity; $F_{\mathrm{ST}}$, Genetic differentiation. 
叶俊伟，田斌 (2021) 中国西南地区重要木本油料植物扁核木的遗传结构及成因. 生物多样性, 29, 16291637.

http://www.biodiversity-science.net/CN/10.17520/biods.2021341

(a)

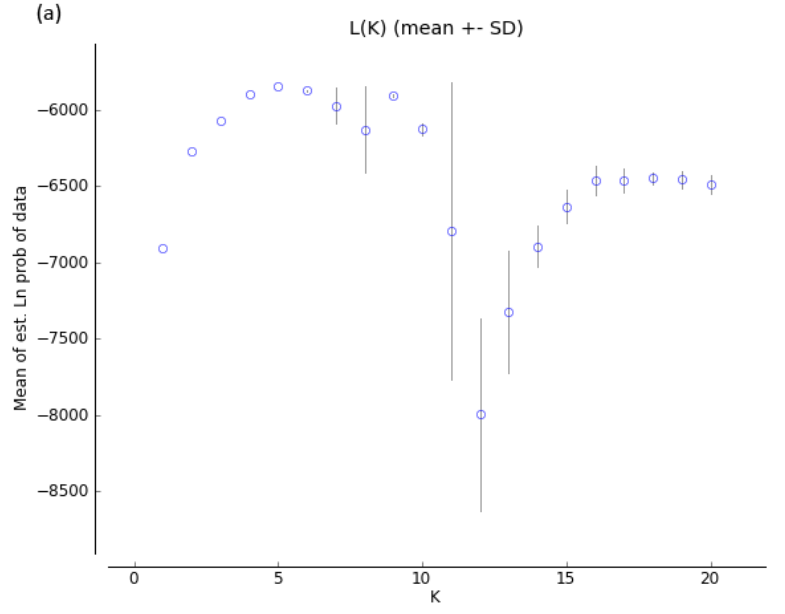

(b)

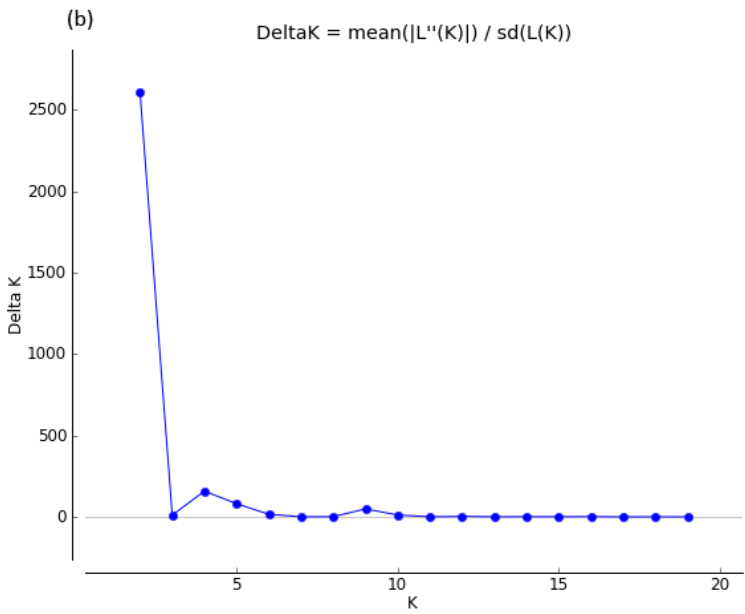

附录3 扁核木32个种群STRUCTURE分析中后验概率 $[\ln P(D)](a)$ 和后验概率的变化速率 $(\Delta K)(b)$ 分布 Appendix 3 Distribution of the change in log-likelihood of the data for each run, $\ln \mathrm{P}(D)$ (a), and $\Delta K$, the second order rate of change of $\ln \mathrm{P}(D)$ between successive $K$-values (b) in STRUCTURE analysis of 32 populations of Prinsepia utilis 
叶俊伟，田斌 (2021) 中国西南地区重要木本油料植物扁核木的遗传结构及成因. 生物多样性, 29, 16291637.

http://www.biodiversity-science.net/CN/10.17520/biods.2021341

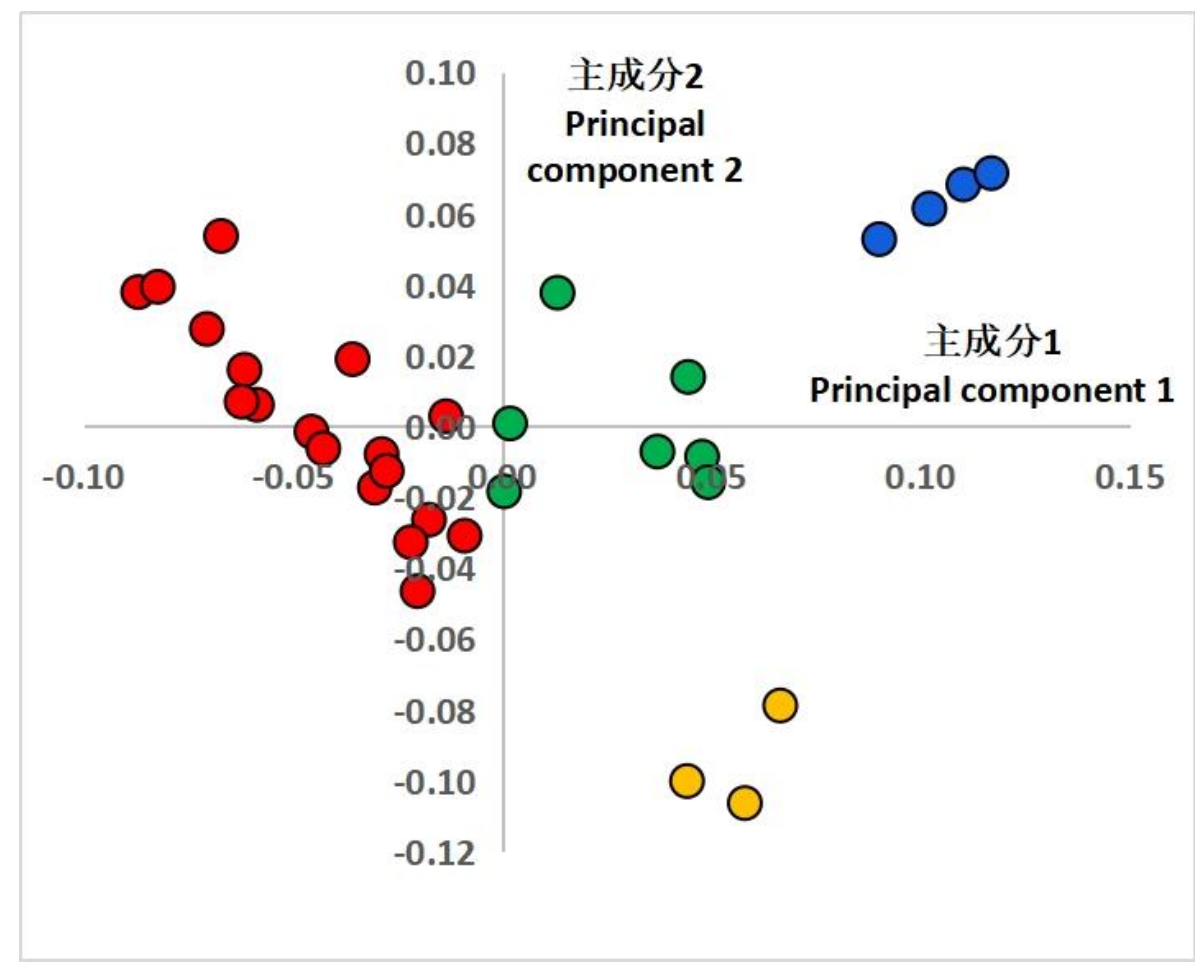

附录4 扁核木32个种群间遗传分化的主成分分析。蓝色为喜马拉雅群组, 绿色为横断山群组, 红色和橙色 分别是云贵高原西部和东部群组。

Appendix 4 Principal component analysis of pairwised genetic differentiation of 32 Prinsepia utilis populations. Blue represents Himalayas group, green represents Hengduan Mountains group, red and orange represent west and east Yunnan-Guizhou Plateau group, respectively. 
叶俊伟，田斌 (2021) 中国西南地区重要木本油料植物扁核木的遗传结构及成因. 生物多样性, 29, 16291637.

http://www.biodiversity-science.net/CN/10.17520/biods.2021341
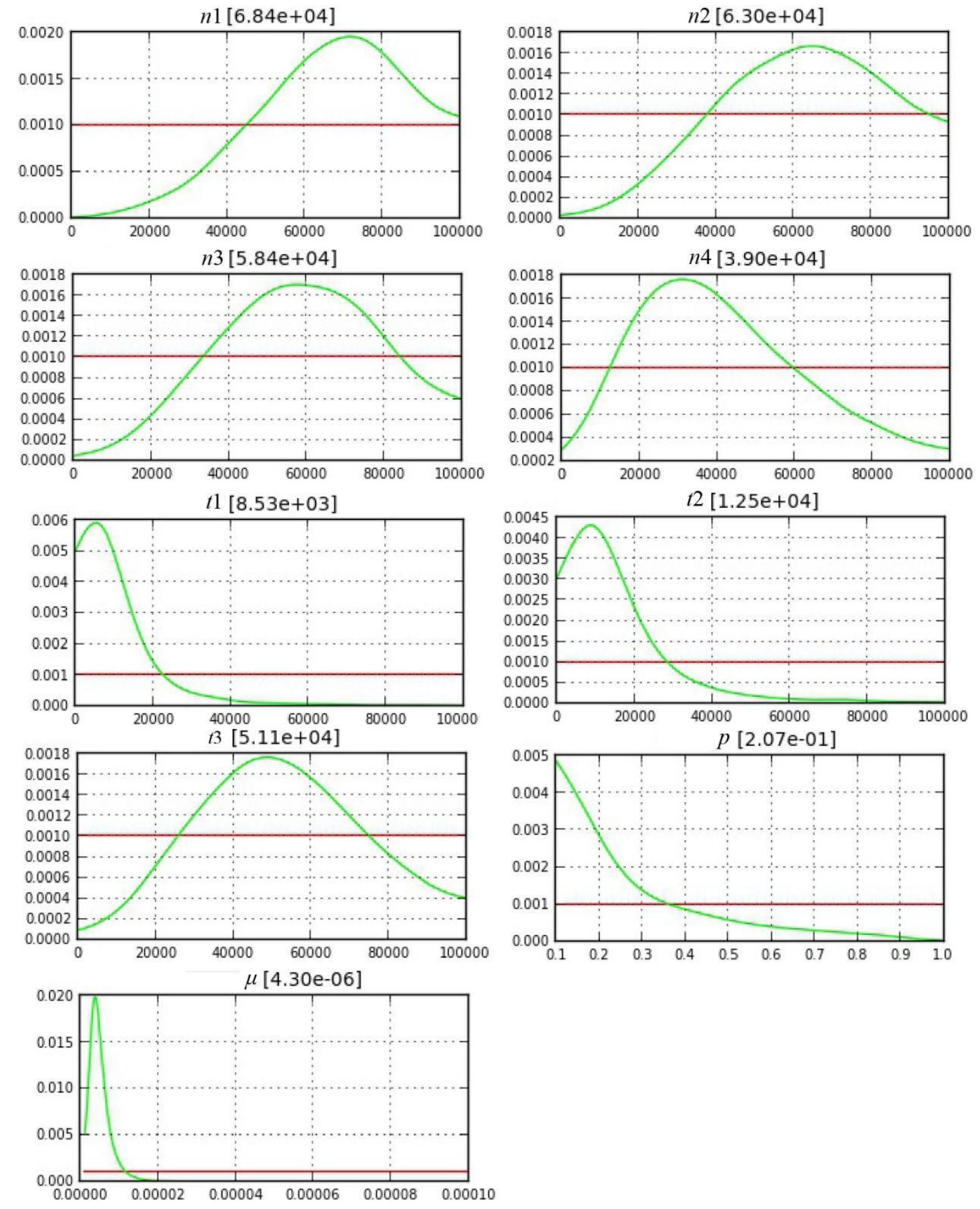

附录 5 DIYABC 分析最优种群分化历史不同参数的先验(红色线条)和后验分布(绿色线条)。 $n 1-4$ : 喜马拉 雅、横断山以及云贵高原西部和东部遗传群组的有效种群大小, $t 1-3$ : 不同种群间分化的分化时间(以世代 为单位), $\mu$ : 突变速率(每位点每世代); $p$ : 广义逐步替代模型中多步突变的比例。

Appendix 5 Prior (red line) and posterior (green line) distributions of different parameters of the most possible divergence scenario of Prinsepia utilis in DIYABC analysis. $n 1-4$ represent effective population sizes of the Himalayas, Hengduan Mountains, west and east Yunnan-Guizhou Plateau genetic clusters, respectively, $t 1-3$ represent divergence times of different divergence events (in generations). $\mu$ : Mutation rate (per generation per locus); $p$, The proportion of multiple step mutations in the generalized stepwise model. 